\section{Building brains from genes}

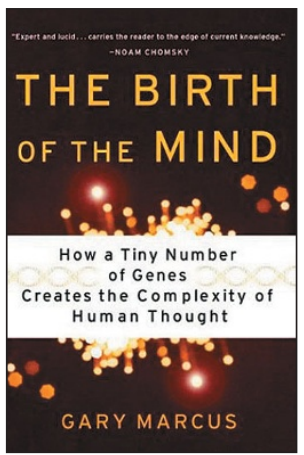

\section{The Birth of the Mind: How a Tiny Number of Genes Creates the Complexities of Human Thought}

by Gary Marcus

Basic Books, 2004

288 pp. hardcover, $\$ 26$

ISBN 0465044050

\section{Reviewed by Charles Jennings}

About half of the estimated 30,000-odd genes in the human genome are expressed in the brain. Among these genes is hidden the explanation for our unique human cognitive abilities, and for many of the differences between individual people. Developmental neurobiology is the essential bridge for connecting genome to behavior, but despite its obvious importance, there has not yet been a popular book devoted to this subject.

The Birth of the Mind is an ambitious attempt to fill this gap. The author, Gary Marcus, is a cognitive scientist, but he has learned a lot about developmental neurobiology and has written a concise and very readable introduction to the field. By drawing on related disciplines such as genetics, cognitive science and evolution, he provides an overview of how the interaction between genome and environment gives rise to the human brain - and by extension the human mind.

Marcus gives as clear an account as I have ever seen of the nature versus nurture 'debate'. In fact, most biologists no longer regard this as a debate (genes and environment are both important), and the fact that it is still perceived as such by the public may reflect the lack of clear popular account, which this book now provides. The concepts of pleiotropy, heritability and the interaction between genes and environment are also clearly explained. The popular press loves to announce the discovery of 'genes for' everything from adultery to zoophilia, and this will probably continue as long as there is a demand for light scientific entertainment; anyone who reads this book, however, will understand why this is a misrepresentation of what genes actually do.

I found the discussion of innate behaviors particularly insightful. These are often described as 'hardwired', the implication being that they are inflexibly determined by the genome. But as Marcus emphasizes, there is a distinction between 'hardwired' and 'prewired'. Just as computer software often comes with default settings that can be customized by the user, so the brain develops with prewired patterns that can be modified by later experience. The failure to distinguish these

Charles Jennings is Executive Editor of the Nature Research Journals.

e-mail: c.jennings@natureny.com two situations has led to endless confusion in the debate between nativism (the belief that cognitive abilities are prewired) and empiricism (the opposite), and Marcus does a nice job of recasting the discussion in a more constructive way.

He also dispels a more recent myth, namely that there is a 'gene shortage' that precludes genes from encoding complex behaviors. It is admittedly surprising that we have only 30,000 genes but 100 billion neurons, particularly given that the nematode C. elegans has nearly as many genes yet only 302 neurons. But as Marcus makes clear, genes are complex individually and give rise to even greater complexity by acting in combination; moreover, the truth is that we have no basis for surprise, absent a theory to explain how many genes are needed for a given degree of biological complexity.

Einstein famously advised that everything should be made as simple as possible, but no simpler. Marcus takes this to heart, and his book contains many simplifications but few misrepresentations. Where to simplify is a matter of taste, and not everyone will agree with his decision to avoid the complexities of transcriptional and posttranscriptional regulation. He compares genes to Boolean logic gates; IF a certain combination of transcription factors is present, THEN make this protein. This is a useful analogy for the general reader, but it may be a violation of Einstein's maxim. Genes sometimes behave as analog rather than digital devices; that is, they show graded rather than on/off expression. An important issue for the emerging field of systems biology is to develop theoretical frameworks for understanding gene interaction networks, and it is not clear whether discrete models will suffice or whether more complex continuous models will be needed. But Marcus's formulation is certainly provocative and probably not seriously misleading for his target audience.

The book is enjoyable to read. Marcus writes with a light touch (his mentor was Steven Pinker, and it shows), and if he occasionally goes too far (e.g., "without genes, learning would not exist", p. 170), an occasional vapidity seems preferable to the ponderous academic prose by which too many scientists express themselves.

I have one reservation, however; despite the book's title, anyone looking for philosophical insights into the mind/brain relationship will be disappointed. Marcus announces this at the outset. Referring to Francis Crick's 'astonishing hypothesis' that our thoughts are determined by physical events within our brains, he says: "I can't say that I am astonished. To many people of my generation, it has become obvious (maybe even banal) that our thoughts are the product of our brains." I wonder whom he has been talking to. In my experience, the great majority of lay people are dualists, and some find the materialist viewpoint profoundly disconcerting. Even those of us who consider ourselves hard-nosed materialists sometimes sense the chasm that separates our scientific beliefs from our sense of self. Marcus seems untroubled by such thoughts, and his book will be of little help to those who are.

But overall, The Birth of the Mind is a fine general introduction and I have no hesitation recommending it to students, scientists from other disciplines, or lay readers wanting to learn something about this fascinating and fast-developing field. 